rheuma plus $2015 \cdot 14: 1$

DOI 10.1007/s12688-015-0023-5

Online publiziert: 13. Februar 2015

๑) Springer-Verlag Wien 2015

\section{Liebe Leserinnen und Leser,}

wieder stehen wir am Beginn eines neuen Jahres. Bisher hat jedes neue Jahr auch Veränderungen von rheuma plus gebracht, für das heurige Jahr 2015 sind derartige Veränderungen nicht in Sicht, obwohl man natürlich niemals wissen kann, was die Zukunft so bringen wird. Der redaktionelle Workflow wurde bereits 2014 entscheidend verändert, und das gesamte redaktionelle Team möchte sich bei den Autoren für ihre Kooperationsbereitschaft und ihr Verständnis in diesem $\mathrm{Zu}$ sammenhang bedanken. Gemeinsam mit den Autoren ist das redaktionelle Team weiter bemüht, die Linie von rheuma plus fortzusetzen und für Sie hoffentlich interessante Aspekte aus dem großen Gebiet der Rheumatologie zu beleuchten.

Diesem Anspruch versucht selbstverständlich auch die erste Ausgabe 2015 Rechnung zu tragen. In einem Praxisbeitrag zeigt Ulrike Stummer, dass das Alter primär nicht als Ausschlusskriterium für Erkrankungen, wie z. B. eine Sklerodermie, heranzuziehen ist. Andererseits ist aber auf alle Fälle ein altersadaptiertes Management angezeigt.

Auch diesmal nimmt die Anwendung biologischer DMARDs einen prominenten Platz in rheuma plus ein. Es ist ein Allgemeinplatz, zu behaupten, dass die Einführung dieser Substanzen die rheumatologische Therapie und andere mehr, wie z. B. die Behandlung der Psoriasis oder entzündlicher Darmerkrankungen, revolutioniert hat. Jetzt gilt es, die Anwendung dieser Präparategruppen zu optimieren und $\mathrm{zu}$ individualisieren. Dazu sind Erkenntnisse aus Registern wie dem BioReg unerlässlich. Zum BioReg bringen wir die redaktionelle Darstellung eines Vortrags von Jochen Zwerina anlässlich der Jahrestagung der ÖGR. Dieser Beitrag zeigt neue Entwicklungen und Erkenntnisse aus dem österreichischen Register, das

Burkhard Leeb

II. Med. Abteilung, LK Stockerau, NOE Kompetenzzentrum für Rheumatologie, Stockerau

\title{
Nur die Veränderung hat Bestand
}

sich weiterhin in sehr erfreulicher Weise positiv entwickelt.

Inhaltlich zum Biologika-Schwerpunkt passt auch der Beitrag von Michael Schirmer, der sich mit therapeutischen Optionen bei der nicht-radiographischen Spondylarthritis befasst. Dieses, vielleicht nicht so einfach abzugrenzende, Krankheitsbild wird aus klinischer Sicht von einem der österreichischen Experten auf diesem Gebiet, nämlich Josef Hermann unter besonderer Berücksichtigung der Abgrenzung zum chronischen Rückenschmerz erläutert.

Auch auf dem ACR-Kongress in Boston 2014 war den Spondylarthritiden breiter Raum gewidmet. Neue therapeutische Optionen, wie z. B. Antikörper gegen IL-17, aber auch „small molecules", wie Apremilast, werden in Hinkunft unser therapeutisches Armamentarium erweitern. Miriam Gärtner zeigt ihre Sicht dieser Highlights in Clinical Science vom ACR 2014. Stephan Blüml stellt die Highlights der Grundlagenforschung vor.

Neben dem großen Interesse an autoimmunologisch bedingten entzündlichrheumatischen Erkrankungen kommt einer alten Bekannten, nämlich der Gicht, wohl auch auf Grund des Lebensstils eines Großteils der Gesellschaft, vermehrt Bedeutung zu. Judith Sautner beleuchtet den Wissensstand der Kollegenschaft unter besonderer Berücksichtigung rezent entwickelter und veröffentlichter Empfehlungen zum Management der Gicht mit einem - das sei vorweggenommen - durchaus positiven Ergebnis.

$\mathrm{Zu}$ alldem passt das Motto „Mit dem Patienten, nicht über ihn hinweg“, das Klaus Machold, der neue Präsident der ÖGR, in seinem programmatischen Interview vorgibt.

Abschließend ergeht, wie immer, die herzliche Einladung an die geschätzte Leserschaft, uns Manuskripte, Fallberichte, Diskussionsbemerkungen, Beiträge zur aktuellen oder zu früheren Ausgaben sowie selbstverständlich auch zu anderen Themen, die Sie für relevant halten, zu schicken. Weiters hoffe ich, dass Sie auch in dieser Ausgabe Interessantes für sich finden werden und bedanke mich schon im Voraus für Kritik, Hinweise, Zustimmung und für jeden anderen Beitrag.

Herzlichst,

Ihr Burkhard Leeb

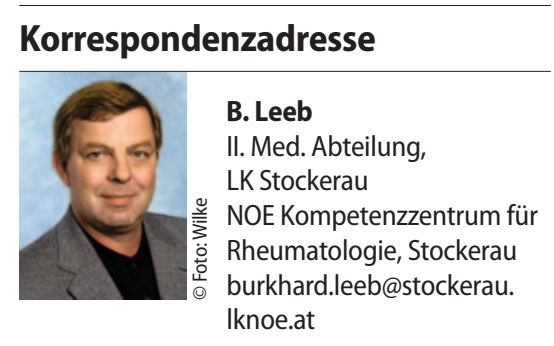

Ordination: Babogasse 20, 2020 Hollabrunn 